\title{
Full Paper \\ A qualitative study of unmet needs and interactions with primary care among cancer survivors
}

\author{
NF Khan", , J Evans' and PW Rose' \\ 'Department of Primary Health Care, University of Oxford, 2nd Floor, 23-38 Hythe Bridge Street, Oxford OX4 IPR, UK
}

\begin{abstract}
INTRODUCTION: Despite increasing numbers, there is little research investigating the long-term needs of cancer survivors. The aim of this study is to explore the experiences of individuals who have survived at least 5 years following a cancer diagnosis, and to describe perceived unmet needs and interactions with primary care.

METHODS: Forty long-term survivors of breast, colorectal and prostate cancer were purposively selected for an in-depth qualitative study. We aimed for a maximum variation sample according to cancer site, gender, time since diagnosis, cancer needs, anxiety and depression. Interviews were audio recorded and transcribed verbatim. Transcripts were coded thematically using a grounded theory approach.

RESULTS: Analysis of the interview data is presented in four subthemes: the role they perceived for the general practitioner (GP), unmet needs, reasons for not using primary care for needs they perceived as cancer related, and ongoing care for cancer-related issues. The majority of cancer survivors did not see a role for their GP in their long-term care related to their cancer diagnosis as most considered that they did not need active follow-up, but some expressed a need for psychological services and information on possible long-term effects. Cancer survivors cited three main reasons for not using GP services in relation to their cancer diagnosis: GPs were seen as non-experts in cancer; they were perceived as too busy; and a lack of continuity within primary care made it difficult to talk about long-term issues. There was a wide variation in schedules and notification of PSA tests among the prostate cancer survivors.

DISCUSSION: The results from this project suggest that some cancer survivors have specific emotional and physical needs that could benefit from input from their primary care team, but not all cancer survivors look to their GP for their long-term cancer-related care. Better information care planning is required from specialists in order to identify those who would benefit most.
\end{abstract}

British Journal of Cancer (20II) I 05, S46-S5I; doi:I0.I038/bjc.20II.422 www.bjcancer.com

(c) 201 I Cancer Research UK

Keywords: neoplasms; survivors; primary health care; qualitative research; general practice

There are at present over 1.2 million cancer survivors living at least 5 years past a cancer diagnosis (Maddams et al, 2009). General practitioners (GPs) are responsible for the majority of health-care needs in this population after the completion of hospital-based monitoring, which is typically between 3 and 5 years post diagnosis. The time following completion of specialist monitoring has previously been described as a 'black hole' when patients may feel abandoned at a vulnerable time (Kendall et al, 2006). There is currently a dearth of literature investigating individual cancer survivors' experience of primary care service use and unmet needs following the completion of specialist monitoring (Khan et al, 2008). As the population of cancer survivors grows and with increasing focus on shorter periods of hospital-based monitoring for cancer patients, it is important to understand the use and quality of primary care services, together with how the role of primary care is perceived within this population (Department of Health, 2010). The aims of this work are to investigate how cancer survivors use GP services, and to identify both unmet needs and the current role of primary care in the care of this population.

*Correspondence: Dr NF Khan; E-mail: nada.khan@phc.ox.ac.uk

\section{MATERIALS AND METHODS}

\section{Sample}

An iterative and purposive sample of 40 interviewees was selected from respondents to a linked survey of breast, colorectal and prostate cancer survivors at least 5 years post diagnosis (Harrison et $a l, 2011)$. The survey was conducted within the population covered by the Oxford Cancer Intelligence Unit and Northern and Yorkshire Cancer Registry and Information Service. Interviewees were selected with the aim of achieving maximum variation on the basis of tumour site, age, gender, geographical location, time since diagnosis, and responses to questionnaire subscales on depression, anxiety and cancer-related needs.

\section{The interviews}

All interviews were conducted in the interviewees' home between October 2009 and August 2010 by NK. Each interview started with an open-ended question, inviting the interviewee to tell their story of living past a cancer diagnosis, and then continued with a semistructured approach based on an interview schedule. The interview schedule focussed on the use of primary care services and other 
Box I Major themes from interviews with long-term cancer survivors

Themes relating to health-services use
Cancer follow-up care
Discharge from hospital care
Interactions with primary care
Information needs
Treatment descriptions
Long-term physical and practical effects of cancer
Lifestyle changes as a result of cancer
Physical long-term effects of treatment
Practical issues
Recurrence of cancer
Unmet needs
Long-term feelings and impact on relationships
Feelings about cancer in the long-term
Impact on relationships
Support needs
Other issues not coded against the major themes listed above

survivorship issues previously identified in the literature. All interviews were audio recorded.

\section{Transcribing and analysis}

The digital audio recording of each interview was transcribed verbatim. All interviews were coded thematically using NVivo 7 qualitative analysis software (Doncaster, Australia) by one researcher (NK). Most thematic categories were labelled using descriptive terms grounded in the narratives, whereas others were driven by questions on the interview schedule. The coding categories were developed using the constant comparison method to relate the commonalities and differences between each individual narrative (Glaser, 1965). This method involved reading the transcripts and coding sections of each transcript to common coding categories, or themes, which linked ideas from the different narratives together. A second researcher (JE) reviewed and commented on the coding categories.

In total, the interview data were coded against 13 major themes (see Box 1). Following initial coding of the interviews, selected major themes were examined using the 'One Sheet of Paper' (OSOP) method (Ziebland and McPherson, 2006). This secondary and more detailed method of coding facilitated the recognition of patterns and commonalities between the respondents. Researchers NK and JE independently produced OSOPs for the major themes classified as 'interactions with primary care' and 'unmet needs'. Subthemes on the OSOPs were discussed, developed and organised according to how the respondents to this study used primary care services.

\section{RESULTS}

Forty long-term survivors of breast, colorectal and prostate cancer were interviewed for this study (see Appendix for details). A summary of the respondents' characteristics is shown in Table 1. Reflecting national trends in cancer prevalence, most respondents were aged between 61 and 80 years at the time of interview. Themes relating to patient interactions with primary care were organised and are presented in four subthemes: the perceived role of the GP, unmet needs, reasons for not using primary care for cancer-related needs and ongoing care for cancerrelated issues.

\section{Role of the GP in long-term care}

The majority of cancer survivors did not see a substantial role for their GP in their long-term cancer care. Although these
Table I The interview sample

\begin{tabular}{lcccc}
\hline Total number & $\begin{array}{c}\text { Breast } \\
\mathbf{1 5}\end{array}$ & $\begin{array}{c}\text { Colorectal } \\
\mathbf{1 3}\end{array}$ & $\begin{array}{c}\text { Prostate } \\
\mathbf{1 2}\end{array}$ & $\begin{array}{c}\text { Total } \\
\mathbf{4 0}\end{array}$ \\
\hline Gender & & & & \\
Male & 0 & 6 & 12 & $\mathbf{1 8}$ \\
Female & 15 & 7 & 0 & $\mathbf{2 2}$ \\
Location & & & & \\
OCIU & 10 & 10 & 6 & $\mathbf{2 6}$ \\
NYCRIS & 5 & 3 & 6 & $\mathbf{1 4}$ \\
Age (in years) & & & & \\
60 or younger & 5 & 3 & 0 & $\mathbf{8}$ \\
$61-70$ & 5 & 3 & 2 & $\mathbf{1 0}$ \\
$7 \mid-80$ & 5 & 5 & 6 & $\mathbf{1 6}$ \\
81 and above & 0 & 2 & 4 & $\mathbf{6}$ \\
& & & & \\
Length of survival (years) & & 8 & 8 & $\mathbf{2 4}$ \\
$5-7$ & 8 & 2 & 3 & $\mathbf{7}$ \\
$8-1 \mid$ & 2 & 3 & 1 & $\mathbf{9}$ \\
12 and above & 5 & & & \\
\hline
\end{tabular}

Abbreviations: NYCRIS = Northern and Yorkshire Cancer Registry and Information Service; OCIU = Oxford Cancer Intelligence Unit.

respondents acknowledged opportunities for directly accessing GP services, most spoke of cancer being 'in the past', and assessed themselves as healthy individuals not requiring active monitoring. When asked whether there was anything else that his GP could be doing for him in terms of his cancer-related care, one participant stated, 'No, not at all. I think it's in the past now and what's happened has happened... what's done is done and that's it really' (patient identifier 05, prostate cancer). Another supported this view, noting that 'I haven't gone [to see my GP about cancer related issues] for quite a long time because I don't feel any adverse effect' (patient identifier 31, prostate cancer).

Primary care services were generally perceived as easy to access, and some GPs had encouraged their patients to contact them with any ongoing problems. One participant stated that he felt 'they're [the GP] there and I can go any time... I could be up there and be attended to' (patient identifier 15, male colorectal cancer) and another described how his doctor had told him 'if you've got any problems at all, an ache here, an ache there, just come in...even if you just want to talk' (patient identifier 23, male colorectal cancer).

\section{Unmet needs}

In contrast to those who felt that there was little their GP could do for them, other respondents spoke about unmet needs that they felt could be addressed in primary care. Several survivors expressed a desire for ongoing psychological counselling, which was not offered by either hospital or primary care teams. One breast cancer survivor felt that there was more her GP could have done to deal with her ongoing depression:

\section{8, Breast cancer}

I: Do you think that there was something that [your GP] could have been doing to help you?

$\mathrm{R}$ : I think it could have been looked into more really, because I have wondered whether it was depression, because when I've read about symptoms of depression I've thought, 'Well, yes. That's how I feel.' And I did wonder whether there was a depression element to it although I don't sit around feeling miserable as such or weeping. But I did wonder that and it might have been helpful to sit down with a doctor and really talk through it. And just see whether there was something that 
could be done or whether it was something I was just going to have to adapt to and find coping strategies with.

A few respondents spoke about the lack of availability of complementary and alternative therapies within primary and secondary care during and after the treatment. Those who accessed complementary techniques such as Reiki, hypnotherapy, Bowen technique, Shiatsu and acupuncture noted regional variations in the provision of these services on the NHS, which prompted some respondents to travel to specialist centres. This colorectal cancer survivor who felt that she benefitted greatly from using Shiatsu describes not being able to access this complementary therapy through her GP surgery or through the NHS:

\section{4, Female colorectal cancer}

It seems sensible for the NHS to utilise complementary therapy and I mean complementary is the word here. And they were available in London but not in [respondent's local town] so that was a bit disappointing. So I had to ask around and try and get help from contacts and so on.

Finally, some respondents expressed a need for more information during their long-term care, particularly relating to late effects of cancer treatment. A colorectal cancer survivor felt that he was not ready to receive information related to bowel dysfunction immediately following surgery because 'so many things happen... you're depressed and you're worried... you listen but you don't take it all in' (patient identifier 22, male colorectal cancer). Another breast cancer survivor echoed this sentiment, saying 'the average person could do with more information...there was a pack I came away with from the hospital. I never read it... it has to be at the right time. When you come out of hospital all you want to say is, 'I'm out of hospital'. You don't want to read about it and anyway, you're too tired' (patient identifier 27, breast cancer survivor).

\section{Reasons for not using primary care services for cancer-related needs}

Certain individuals had chosen not to discuss their cancer-related concerns with a GP. The three main reasons given were that GPs were seen as non-experts in cancer, were too busy to be 'bothered' with cancer-related issues and, lastly, that a lack of continuity in primary care hindered discussions relating to cancer and its longterm effects.

GPs are not experts in cancer The first reason is that many respondents expressed the opinion that GPs were not sufficiently trained or experienced to provide cancer-related follow-up care. These long-term survivors preferred to discuss cancer-related issues with a cancer consultant, despite having been released from hospital-based monitoring. Two men echoed this sentiment:

\section{8, Male colorectal cancer}

The GP, they are only a first port of call aren't they, because ... if there's something really wrong with you they will pass you on to a specialist. They won't deal with it themselves. They're not like the old-fashioned family doctor that had to do the lot... I think it's a good thing because, after all, they can't be experienced in every disease there is.

\section{3 , prostate cancer}

A GP has got to be a generalist by nature...I think when something is potentially life threatening you would like to feel a specialist is giving you the answers as opposed to a generalist, although some generalists are very good.

GPs are too busy to be bothered with long-term cancer-related problems A second reason for not accessing primary care services was an impression that GPs were too busy, or that GPs would view long-term cancer-related worries as minor complaints not worth discussion. One woman felt that 'you don't want to keep bothering the GP' (patient identifier 03, breast cancer). Some survivors noted that although their GP was busy, there were opportunities for short discussions about cancer-related issues within the course of another appointment. A long-term colorectal cancer survivor thought that 'the doctors could bring the subject up in conversation with men... when they're visiting them for other reasons' (patient identifier 20, male colorectal cancer).

Lack of continuity of care A third reason for not using GP services for cancer-related care was a lack of continuity within some primary care practices. Respondents in practices without a named GP spoke about their frustration at not being able to see the same GP from visit to visit. One interviewee discussed the high turnover of staff in his primary care practice, which triggered him to bypass his GP and go straight to his cancer consultant for his follow-up appointments:

\section{4 , prostate cancer}

One of the problems is the doctor that diagnosed me in the first place moved on a long time ago. He was a nice enough chap. You could talk to him... but he didn't stay long and the next doctor came in and it coincided... with a change in the general running of the surgery. It's quite a big surgery. There are about fifteen doctors there. It's like going into a mini hospital... they ask you questions and they haven't got a clue and they obviously haven't gone back over the records. I've complained to the surgery and I've said to them, 'Look, I think it's important in any history with a patient and a doctor that the doctor has some knowledge of what's been going on in the past, preferably through knowing them. But if you keep swapping people about they've got no idea.' And you move to the next doctor and you've got to start all over again and tell them everything again and I find that this is not terribly good. And so this is why I go to the consultant [directly]. When I get there he opens his file up and... he knows me and I'm happy with that.

These cancer survivors noted the challenge of discussing cancerrelated issues with a GP who did not have the correct notes or information about their cancer or was unaware of the previous cancer diagnosis before a consultation.

\section{Ongoing care for cancer-related issues}

Respondents emphasised the value of their GP briefly following up on their cancer from time to time to assess their recovery. Although most felt that formal follow-up through primary care was unnecessary, many noted that there were opportunities for informal care during the course of appointments for other health issues. A long-term colorectal cancer survivor noted that 'the doctors could bring the subject up in conversation with men... when they're visiting them for other reasons' (patient identifier 20, male colorectal cancer). Some wanted a more active but quick check, with the suggestion that a 'follow-up phone call once a year would be quite nice' (patient identifier 07, prostate cancer).

There was some variation among these long-term cancer survivors in terms of whom they would contact with a cancerrelated question or concern. Many acknowledged the gate-keeping role of primary care. A number of respondents agreed that although they would initially contact their GP with any queries, it was reassuring to have a direct contact at the hospital as well. Some were given contact details for a cancer nurse; for instance, a breast cancer survivor describes how 'when I left she [the breast care nurse] gave me her card and she said she'd always be in touch'. This respondent continued to say that she would initially contact the breast care nurse who 'could direct me to whoever I needed to talk to' (patient identifier 06, breast cancer). One patient was 
advised to approach secondary care services directly and bypass her GP, 'I think they [her consultants] said, 'If you have any problems in the future come back to us... don't worry about going through your GP' (patient identifier 02, breast cancer). However, not all cancer survivors were provided with direct contact information for their cancer consultant or clinical nurse specialist.

There was wide variation surrounding the procedures and notification of PSA testing. Many prostate cancer survivors wanted more information on thresholds for starting second-line treatment and notification of test results. Feedback of test results was often lacking, as one man pointed out, 'My doctor has never got those [PSA test] results and contacted me to say, 'Come down and we'll have a talk.' No...none of them has ever done that. It may have been a nice thing to have done to give me a bit more confidence' (patient identifier 38, prostate cancer). Prostate cancer survivors who received PSA test results during their hospital-based care felt that this service should continue, 'I would like I'd like a little letter like the hospital used to do because it's always is it [the PSA level] going up or is it coming down.' (patient identifier 40, prostate cancer).

\section{DISCUSSION}

\section{Summary of main findings}

Because of the growing number of cancer survivors in the UK and a trend towards shorter periods of hospital-based monitoring, primary care will have an increasingly important role in the ongoing care of long-term cancer survivors. Although the majority of cancer survivors in this study talked about 'moving on' from their previous diagnosis and did not perceive that they had any problems requiring active primary care involvement, some cancer survivors had ongoing needs including information on psychological services and late effects of treatment. Although primary care was seen as accessible, some were hesitant to consult with their GP for cancer-specific matters due to a concern about lack of GP expertise with oncological issues, or a perception that GPs were 'too busy'. Participants noted deficiencies in the primary care systems, in particular notification around PSA test results.

\section{Comparison with previous literature}

Cancer survivors have previously identified lack of oncology expertise as a potential barrier to accessing primary care follow-up services (Kantsiper et al, 2009; Adams et al, 2011). Primary care providers in a US-based study reflected this view, with a preference for cancer survivors to continue specialist follow-up (Kantsiper et al, 2009). However, developments in guidance for cancer followup services mean that primary care will increasingly take on the responsibility for cancer follow-up at an earlier stage in the UK (National Institute for Health and Clinical Excellence, 2002). Participants in our study reported a wide range of preferences regarding whom to contact following completion of routine hospital follow-up. Previous work suggests that cancer patients who are told to contact their GP by hospital staff at the end of the hospital monitoring phase are significantly more likely to do so than those who were not, suggesting room for improvement in the effective sign-posting of primary care-based services for cancer survivors (Allgar and Neal, 2005).

We found a wide variation in PSA follow-up practices among the long-term cancer survivors in this study. These findings were mirrored in a recent report of the experiences of UK prostate cancer patients. In their study, O’Brien et al (2010) describe the practice of only contacting men with abnormal PSA test results as concerning to those used to regular feedback during hospital follow-up . Notification and information on PSA test results were both significant to the prostate cancer survivors in our study because it represented tangible evidence of their cancer remission. The UK National Cancer Survivorship Initiative has emphasised the importance of developing a clear plan for cancer monitoring tests with clearly understood prompts for action, and it appears that this is currently with relation to PSA test results within primary care (NHS Improvement, 2011).

Although there is little previous research relating to the individual experience of using primary care services among cancer patients, cancer is increasingly viewed as a chronic disease (Beyer, 1995; Hewitt et al, 2006; Ganz, 2009) and therefore one that is suited to management in primary care. It is possible to draw parallels between the experiences of cancer survivors and those who are living with other long-term health conditions. Although the use of the primary care model for chronic diseases such as diabetes and congestive heart failure is associated with improved patient outcomes, this model is not currently applied to long-term cancer survivors (Bodenheimer et al, 2002c; 2006). The chronic care model is based on an understanding that the majority of chronic illness care is performed in the primary care setting (Bodenheimer et al, 2002b). A main focus of this model involves increasing the emphasis on self-management support, whereby patients themselves become the principal caregivers. People who are living with chronic diseases are encouraged to work with their primary care team to acquire the skills to manage their chronic disease by routinely assessing and solving their own problems with the appropriate information (Bodenheimer et al, 2002a). An initial appointment with a GP at the end of hospital-based monitoring to discuss signs and symptoms of depression, late effects of treatment and information needs may facilitate this shift towards supportive self-management within the population of long-term cancer survivors, some of whom will not be aware of the long-term implications of living past cancer and its treatment (Adams et al, 2011).

\section{Implications for practice and policymakers}

Although the majority of long-term cancer survivors do not require ongoing monitoring for cancer-related issues after 5 years, a proportion face long-term cancer-related unmet needs. High-risk groups may include those patients who are experiencing depression and those with additional comorbid diseases (Hewitt et al, 2003; Foster et al, 2009). Many cancer survivors in this study suggested that it might be helpful for the GP to briefly raise the previous cancer in the course of regular care for other conditions. This could satisfy the need to have their cancer experience acknowledged, provide an opportunity to discuss any concerns they had and provide reassurance. Cancer survivors may benefit from a short appointment with a member of their primary care team at the end of hospital follow-up to discuss psychological issues, information on late effects of treatment and how to seek help with any ongoing concerns. Inviting patients diagnosed with cancer for a specific cancer care review could provide cancer survivors with the reassurance that they can talk about cancerrelated issues without the worry that they are 'bothering' their GP (Adams et al, 2011).

Reporting of PSA test results was not standardised across the country. Some prostate cancer survivors were concerned when they did not receive these results from their primary care followup; however, most acknowledged that this probably meant that their results were within normal limits. GPs caring for prostate cancer survivors should manage patient expectations by explaining whether PSA test results will be shared with the patient, and discuss thresholds for restarting treatment.

Continuity of care was identified as an important aspect of primary care-based follow-up by some of the cancer survivors in this study. Previous research has shown that patients prefer to see the same doctor when dealing with long-term or complex problems, which describes the issues facing many long-term cancer survivors (Tarrant et al, 2003). Greater flexibility in 
appointment-based systems may improve the experience of continuity of care among cancer survivors.

\section{Strengths and limitations}

There are several strengths and limitations to this study. The strengths include the large sampling frame and the in-depth nature of the interviews. The interview sample covered a wide range of experiences and represented people from 5 to 22 years post diagnosis. However, only respondents from a cancer survivor's questionnaire based in the areas covered by two UK cancer registries were eligible to take part in the interviews. Although these two regions cover a diverse range of socioeconomic and urbanised and rural areas, the primary care experiences of the respondents may be different from other regions. Despite efforts to recruit participants from minority ethnic groups, these were not represented in our study.

\section{CONCLUSION}

The results from this project suggest that some cancer survivors have specific emotional and physical needs that could benefit from inputs from the primary care team, but not all cancer survivors feel that they need active long-term cancer-related care from their GP. Some cancer survivors may benefit from increased continuity of primary care systems and more information and feedback on PSA testing to monitor recurrence.

\section{ACKNOWLEDGEMENTS}

We acknowledge the time and contributions of the interviewees. We would also like to thank Judith Beckett for her meticulous and accurate transcription. This article is sponsored by Macmillan Cancer Support through its Research Capacity Development Programme and by Cancer Research UK grant number C23140/ A8854.

\section{Ethics approval}

This work was reviewed by the Oxfordshire Research Ethics Committee B (REC reference number 07/Q1605/61), and approval was given on 25 June 2007.

\section{REFERENCES}

Adams E, Boulton M, Rose P, Lund S, Richardson A, Wilson S, Watson E (2011) Views of cancer care reviews in primary care: a qualitative study. Br J Gen Pract 61(585): 173-182

Allgar VL, Neal RD (2005) General practictioners' management of cancer in England: secondary analysis of data from the National Survey of NHS Patients - Cancer. Eur J Cancer Care 14(5): 409-416

Beyer DA (1995) Cancer is a chronic disease. Nurse Pract Forum 6(4): 201-206

Bodenheimer T, Lorig K, Holman H, Grumbach K (2002a) Patient selfmanagement of chronic disease in primary care. JAMA 288(19): 2469-2475

Bodenheimer T, Wagner EH, Grumbach K (2002b) Improving primary care for patients with chronic illness. JAMA 288(14): 1775-1779

Bodenheimer T, Wagner EH, Grumbach K (2002c) Improving primary care for patients with chronic illness: the chronic care model, Part 2. JAMA 288(15): 1909-1914

Department of Health, Macmillan Cancer Support, and NHS Improvement (2010) National Cancer Survivorship Initiative: Vision. Department of Health, Crown Copyright: London. Report

Foster C, Wright D, Hill H, Hopkinson J, Roffe L (2009) Psychosocial implications of living 5 years or more following a cancer diagnosis: a systematic review of the research evidence. Eur J Cancer Care 18(3): $223-247$

Ganz PA (2009) Quality of care and cancer survivorship: the challenge of implementing the institute of medicine recommendations. J Oncol Pract 5(3): $101-105$

Glaser BG (1965) The constant comparative method of qualitative analysis. Social Prob 12(4): 436-445

Harrison SE, Watson EK, Ward AM, Khan NF, Turner D, Adams E, Forman D, Roche MF, Rose PW (2011) The primary health and supportive care needs of long-term cancer survivors. J Clin Oncol 29(15): 2091-2098

Hewitt M, Greenfield S, Stovall E (eds) (2006) From Cancer Patient to Cancer Survivor: Lost in Transition. The National Academies Press: Washington DC

Hewitt M, Rowland JH, Yancik R (2003) Cancer survivors in the United States: age, health, and disability. J Gerontol A Biol Sci Med Sci 58(1): 82-91
Kantsiper M, McDonald EL, Geller G, Shockney L, Snyder C, Wolff AC (2009) Transitioning to breast cancer survivorship: perspectives of patients, cancer specialists, and primary care providers. J Gen Intern Med 24(Suppl 2): S459-S466

Kendall M, Boyd K, Campbell C, Cormie P, Fife S, Thomas K, Weller D, Murray SA (2006) How do people with cancer wish to be cared for in primary care? Serial discussion groups of patients and carers. Fam Pract 23(6): $644-650$

Khan NF, Ward A, Watson E, Austoker J, Rose PW (2008) Long-term survivors of adult cancers and uptake of primary health services: a systematic review. Eur J Cancer 44(2): 195-204

Maddams J, Brewster D, Gavin A, Steward J, Elliott J, Utley M, Moller H (2009) Cancer prevalence in the United Kingdom: estimates for 2008. Br J Cancer 101: $541-547$

National Institute for Health and Clinical Excellence (2002) Guidance of Cancer Services: Improving Outcomes in Breast Cancer. NICE: UK. Report

NHS Improvement (2011) Effective follow up: testing risk stratified pathways. http://www.ncsi.org.uk/wp-content/uploads/RiskStratifiedPathwaysMay-2011.pdf (accessed at 7 September 2011)

O'Brien R, Rose PW, Campbell C, Weller D, Neal RD, Wilkinson C, Watson EK (2010) Experiences of follow-up after treatment in patients with prostate cancer: a qualitative study. BJU Int 106(7): 998-1003

Tarrant C, Windridge K, Boulton M, Baker R, Freeman G (2003) How important is personal care in general practice? BMJ 326(7402): 1310

Ziebland S, McPherson A (2006) Making sense of qualitative data analysis: an introduction with illustrations from DIPEx (personal experiences of health and illness). Med Educ 40(5): 405-414

cC)(ㄱ) This work is licensed under the Creative Commons Attribution-NonCommercial-Share Alike 3.0 Unported License. To view a copy of this license, visit http://creativecommons. org/licenses/by-nc-sa/3.0/ 
Appendix

Participants in the cancer survivors interview study

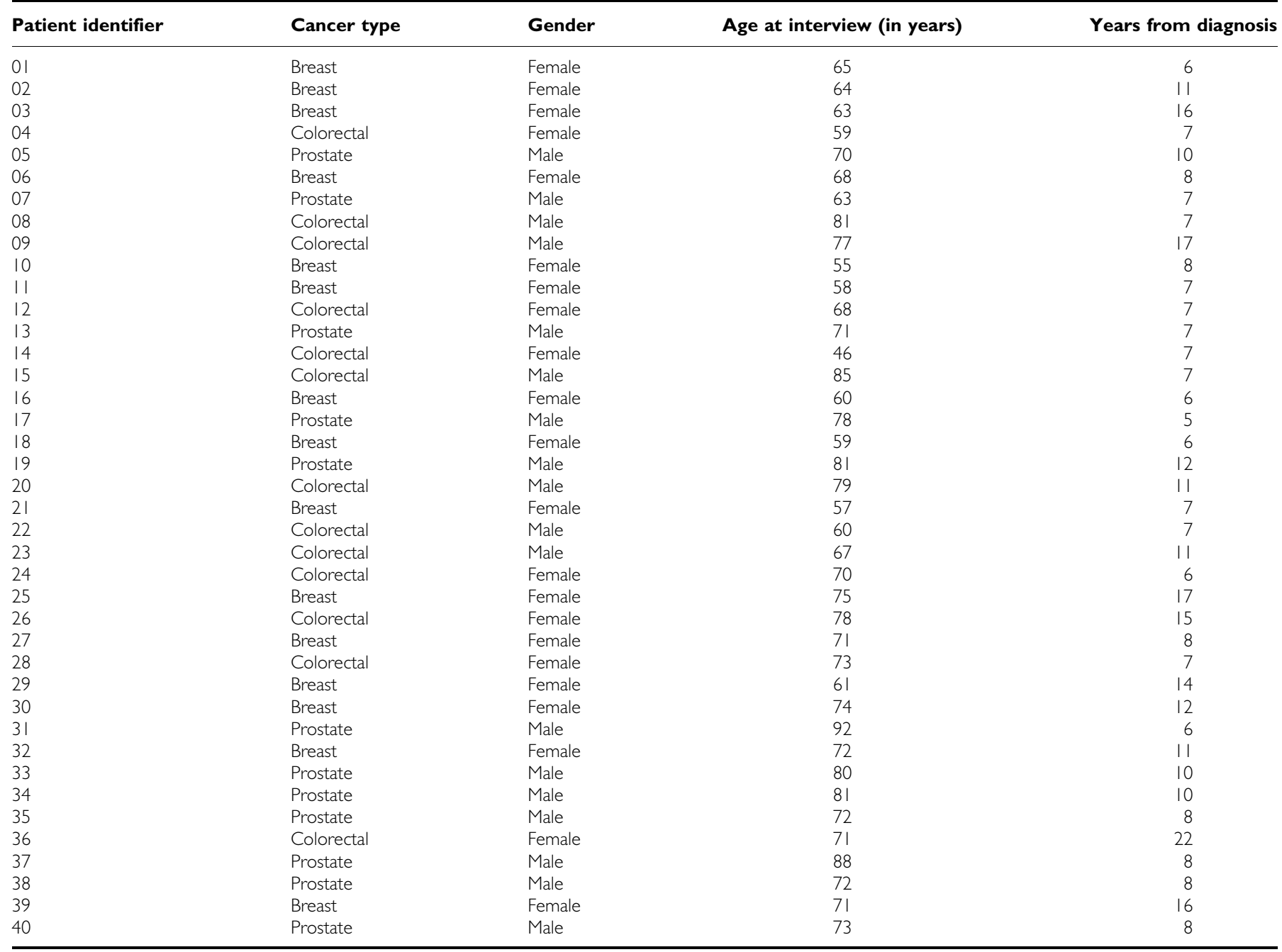

